\title{
Rabies virus is not cytolytic for rat spinal motoneurons in vitro
}

\author{
Céline Guigoni and Patrice Coulon \\ Groupe d'Etude des Réseaux Moteurs, CNRS, Marseille, France
}

\begin{abstract}
Cultures of purified rat embryonic spinal cord motoneurons were used to investigate the capacity of the neurons to survive rabies virus infection in vitro. In crude primary spinal cord cultures, neurons did not survive more than 2 days after rabies virus infection with the fixed strain Challenge Virus Standard. In contrast, virus-infected purified motoneurons resisted cytolysis for at least 7 days, as also did infected motoneurons treated with conditioned medium sampled from rabies virus-infected crude spinal cord cultures. This survival rate was also observed when motoneurons were grown in the presence of astrocytes or fibroblasts and it was not dependent on the presence of growth factors in the culture medium. Moreover, terminal deoxynucleotidy 1 transferase-mediated dUTP nick end labeling experiments showed that only $30 \%$ of infected motoneurons were apoptotic after 7 days of infection. In vivo, despite the massive infection of the spinal cord in infected rat neonates, the moderate number of apoptotic cells in the ventral horn suggests that only a few motoneurons were affected by this mechanism of cell death. Morphometric analyses showed that motoneurons' axon elongated at a comparable rate in virus-infected and noninfected cultures, a sign of high metabolic activity maintained in rabies virus-infected motoneurons. In contrast, hippocampus neurons were susceptible to rabies virus infection, because $70 \%$ of infected neurons were destroyed within 3 days, a large proportion of them being apoptotic. These experiments suggest that spinal cord motoneurons consist in a neuronal population that survive rabies virus infection because the viral induction of apoptosis is delayed in these neurons. They suggest also that paralyses frequently observed in rabid animals could be the consequence of dysfunctions of the locomotor network or of the spinal cord motoneurons themselves, whose parameters could be studied in vitro. Journal of NeuroVirology (2002) 8, 306-317.
\end{abstract}

Keywords: neurotropism; embryonic neurons; hippocampus; apoptosis; quantitative morphometry

\section{Introduction}

Rabies virus (RV) is a neurotropic virus that is responsible for fatal encephalomyelites in mammals. This

Address correspondence to Patrice Coulon, Unité de Neurocybernétique Cellulaire, CNRS, FRE 2102, 280 Bd Sainte Marguerite, 13009 Marseille, France. E-mail: pcoulon@marseille.inserm.fr

This work was supported by the CNRS through the FRE 2102 and ATIPE "Virologie" and the "Université de la Méditerranée." The authors thank C Moretti for his helpful advice in confocal microscopy analysis, M Morel for expert assistance in photography, F Castets for his help in the preparation of astrocyte cultures, C Tuffereau for critical reading of the manuscript, and $\mathrm{M}$ Paul for improving the English style. Special thanks are due to J-P Ternaux for constant support and for fruitful discussions. Part of this work was presented at the 30th annual meeting of the Society for Neuroscience (abstract no. 90.19).

Received 30 January 2002; revised 23 March 2002; accepted 1 May 2002. enveloped virus belongs to the rhabdovirus family and its genome consists in a nonsegmented, negative strand RNA that encodes five proteins. Three of them (N, P, and L) are associated with the RNA to form the nucleocapsid. One $(\mathrm{M})$ interacts with both the nucleocapsid and the plasma membrane and probably plays an important role in the virion maturation process. The fifth is a type I transmembrane glycoprotein $(G)$, the only external viral protein that govern s the viral tropism (see Wagner and Rose for a review, 1996).

Contamination occurs after a bite and viral particles contained in the saliva of infected animals can infect the nerve endings around the wound. Incubation times can be extremely variable depending on both the animal species and the viral strain. They range from a few days for mice infected with the Challenge Virus Strain [CVS, a laboratory strain fully adapted to the mouse brain (Sacramento et al, 1992)], to several 
months, in the case of humans contaminated with so-called street rabies viruses. Usually, examination s of infected brains do not reveal dramatic histological changes in comparison to the marked neurological symptoms and signs (Murphy, 1977). In experimental infections with CVS, some authors pointed out the remarkable conservation of long-lasting infected groups of neurons despite the advanced state of the infection (Ugolini, 1995). On the basis of these in vivo data, RV can be considered as a noncytolytic virus.

In vivo studies have given basic information concerning the mode of propagation of the virus into the central nervous system (CNS). Anatomical analyses of neuronal nuclei infected by RV following peripheral inoculation have demonstrated that the virus propagated in the CNS across chains of synaptically connected neurons (Kuciera et al, 1985; Astic et al, 1993; Ugolini, 1995; Tang et al, 1999). Generally, it is admitted that RV is mainly transported into neurons by the retrograde axonal flow (Gillet et al, 1986). Experiments performed in appropriate systems have shown that RV could be vehicled efficiently via anterograde transport as well (Lafay et al, 1991). Nevertheless, in vivo experiments could not answer questions related to host-virus interactions at a cellular level. To address these questions, in vitro conditions seem more suitable because the parameters of infection could be controlled as precisely as possible. Because RV infection is largely restricted to neurons, in vitro experiments should be performed in primary neuronal cultures in order to be close to natural infection conditions.

So far, only a limited number of studies have been devoted to in vitro experiments using primary cultures of neurons infected with RV. Nevertheless, they provide valuable information on the infectiou s cycle of RV in its host cell. Experiments were carried out using ganglionic neurons (Tsiang et al, 1983, 1989; Lycke and Tsiang, 1987; Castellano s et al, 1997, 2000) or neurons from CNS (Tsiang et al, 1986, 1991). These cultures were used to study in vitro the infectability of neurons under various infection conditions. More recent data were focused on the early steps of RV infection (Lewis and Lentz, 1998; Lewis et al, 2000) and on rabies-induced apoptosis in hippocampal neurons (Morimoto et al, 1999). In both types of study, neurons were shown to survive no more than 4 days after RV infection.

Because the aims of the in vitro experiments were to study virus-neuron interactions, such interaction s could be analyzed more accurately in primary cultures of homogeneous neuronal populations. In the present study, the infection of spinal cord motoneurons (MNs) with RV was chosen mainly because, in natural infection, these neurons are the first to be infected in the CNS after a bite. MNs could be isolated from rat embryos and maintained in culture up to 2 weeks (Bataillé et al, 1998). These cells were infected with CVS or K4-5, a double antigenic mutant differing from CVS by two amino acids in positions
330 (lysine to asparagine) and 333 (arginine to methionine) of the glycoprotein (Coulon et al, 1998). The results were similar to those obtained during in vivo experiments. After intramuscular inoculation of this mutant into adult mice, no MNs could be infected. In the same way, after 6 days of culturing in vitro, only rare rat embryonic MNs were infected with K4-5, whereas several hundreds of them were infected with CVS (Coulon et al, 1998). Thus, primary cultures of MNs seemed to be a promising tool to study the various steps of the RV cycle in neurons.

This motoneuron culture system was used to investigate the survival capacity of these neurons after RV infection in vitro. Our results showed that MNs could survive RV infection as long as 7 days in vitro, a phenomenon that seems specific to this neuronal population because mortality of hippocampus neurons became massive after 3 days of infection. Correlatively, the majority of RV-infected MNs were not apoptotic compared with hippocampus neurons in which the apoptotic process was induced quickly after infection. Thus, it seems that neurons could survive RV infection for a long time as long as the apoptotic process has not started.

\section{Results}

$R V$ does not kill purified MNs in vitro

During previous infection experiments with CVS, some MNs cultures were kept in order to monitor the neuron survival after RV infection. These cultures were grown in presence of neuronal medium not supplemented with growth factors. Surprisingly, it appeared that the MNs were not affected by CVS infection because no virus-induced cytopathic effect (CPE) was observed in these cultures until the degeneration of the neurons occurred naturally after 5 days (short-term culture, Figure 1A). This observation was in contradiction with previous results that indicated that cultures of embryonic mouse hippocampal neurons did not survive more than 2 or 3 days after RV infection (Morimoto et al, 1999). In order to clarify this question, we monitored the survival of purified MNs cultures following infection with CVS virus, in the most favorable conditions of MNs' survival (i.e., in presence of insert of myoblasts). The percentage of living cells was checked daily in infected and noninfected cultures. This estimation was obtained both by observation of the morphology of the neurons in contrast phase microscopy and by trypan blue exclusion (long-term culture, Figure 1B). We noticed that some neurons were considered as dead using the morphology criterion, yet their cytoplasm was still free of trypan blue (data not shown). The results presented in Figure 1C showed no significant difference in the survival rate between infected and noninfected MNs, at least for 7 days following infection, despite high levels of viral protein synthesis (Figure 1D). The rabies-induced mortality appeared 

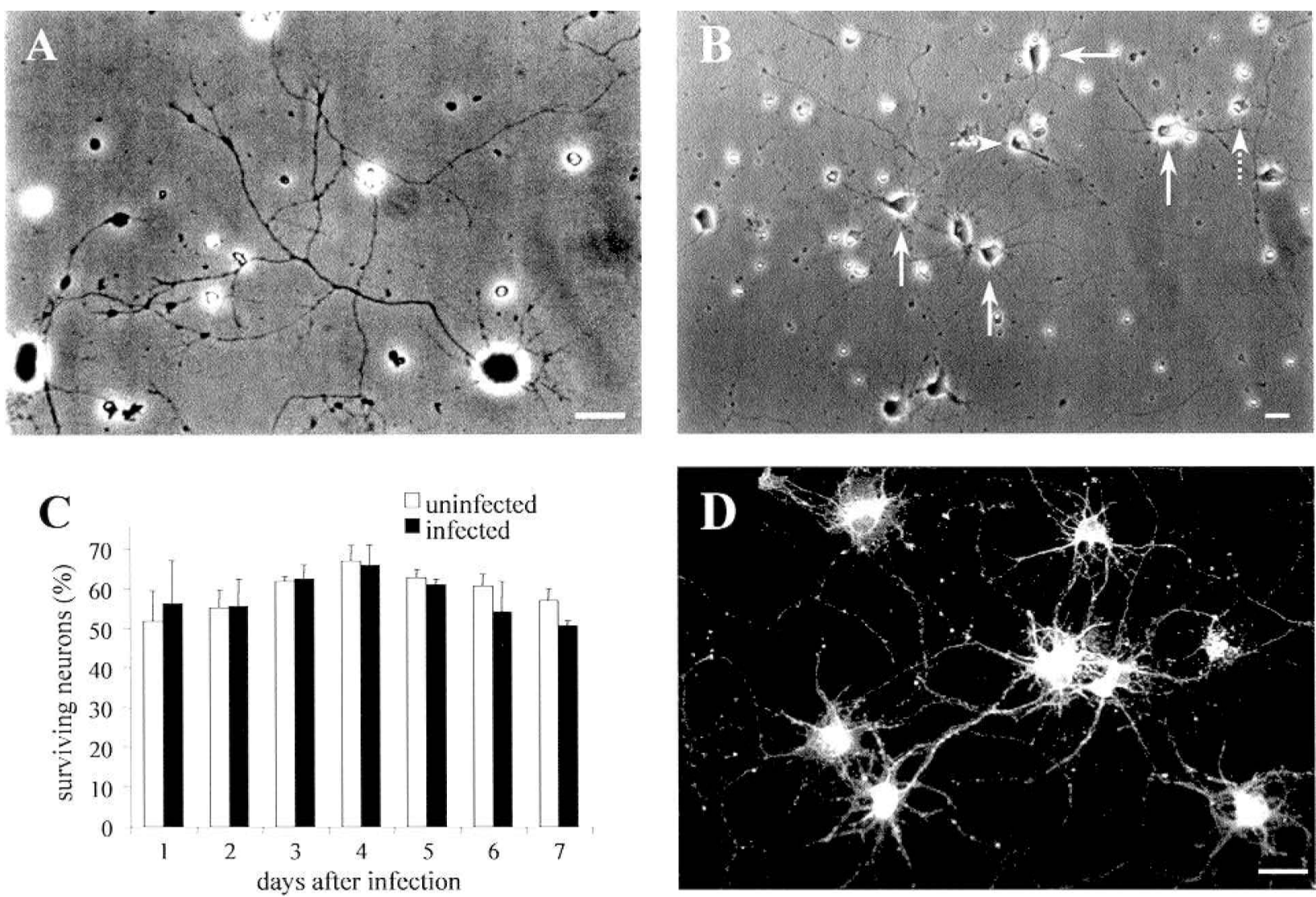

Figure 1 Survival of rat embryonic MNs to RV infection in vitro. (A) Morphology of a MNs infected for 3 days with CVS in a short duration culture. Bar $=20 \mu \mathrm{m}$. (B) The sorting of MNs into dead or living cell categories was estimated from contrast phase pictures taken randomly. In a long-term culture infected for 7 days with CVS, living neurons possessed a dark cell body (full arrows) and dead cells presented a bright inclusion into their soma (dotted arrows). Bar $=20 \mu \mathrm{m}$. (C) The percentage of living neurons in MNs cultures was determined during 7 days following infection with CVS (black bars) or in noninfected control cultures (clear bars). No significant changes were observed. Error bars represent the standard error of the mean. $n=4$. (D) In a long-term culture infected with CVS for 7 days, all MNs were filled with viral antigens. Detection with an anti-P MAb. Confocal image collected as a single optical section after excitation at $543 \mathrm{~nm}$ wavelength. Bar $=25 \mu \mathrm{m}$.

late in the infection process and it was frequently difficult to observe, because of the natural mortality occurring in the cultures after 10 days in vitro.

These experiments were performed using low cell density cultures, i.e., at $1.5 \times 10^{4}$ MNs per coverslip (see materials and methods). In order to test the influence of the cell concentration on the MNs' resistance to RV infection, the survival of infected MNs was checked in high cell density cultures, i.e., at $1.5 \times 10^{5} \mathrm{MNs}$ per coverslip. Under these conditions, infected cultures were maintained for at least 7 days after infection (data not shown) indicating that the cell density did not influence the capacity of MNs to survive RV infection.

\section{Culture conditions influence MNs' susceptibility to $R V$ infection}

All the data previously published about RV infection of neurons in vitro were collected from heterogeneous cultures in terms of cellular populations, i.e., nonneuronal cells were present together with neurons. To compare our data more accurately with those of others, we decided to monitor the survival of RV- infected neurons in crude spinal cord (SC) cultures. In 2-week-old SC cultures, both neurons and glial cells could be detected (Figure 2A). In that case, it was not possible to identify precisely the MNs among the neuronal populations. SC cultures were then infected with CVS and the overall neuronal survival was monitored daily. In cultures infected for $24 \mathrm{~h}$ with CVS, all neurons were heavily infected (yellow labeling in Figure 2B), whereas glial cells were uninfected or contained rarely small nucleocapsid inclusions (yellow labeling indicated by the arrow in Figure 2C). When the infection was allowed to proceed, a strong CPE was observed in the culture as early as 2 days p.i. This CPE mainly concerned neurons and it was confirmed by a dramatic drop in the number of $\mathrm{MAP}^{+}$cells. More than $70 \%$ of neurons disappeared from the cultures (Table 1). In contrast, a large number of cells were still GFAP ${ }^{+}$(data not shown), indicating that glial cells did not seem to be affected by RV-induced CPE in vitro. Beyond 3 days p.i., there were no more MAP2 ${ }^{+}$cells in infected cultures.

To test the influence of exogenous factors on the survival of purified MNs following RV infection, 

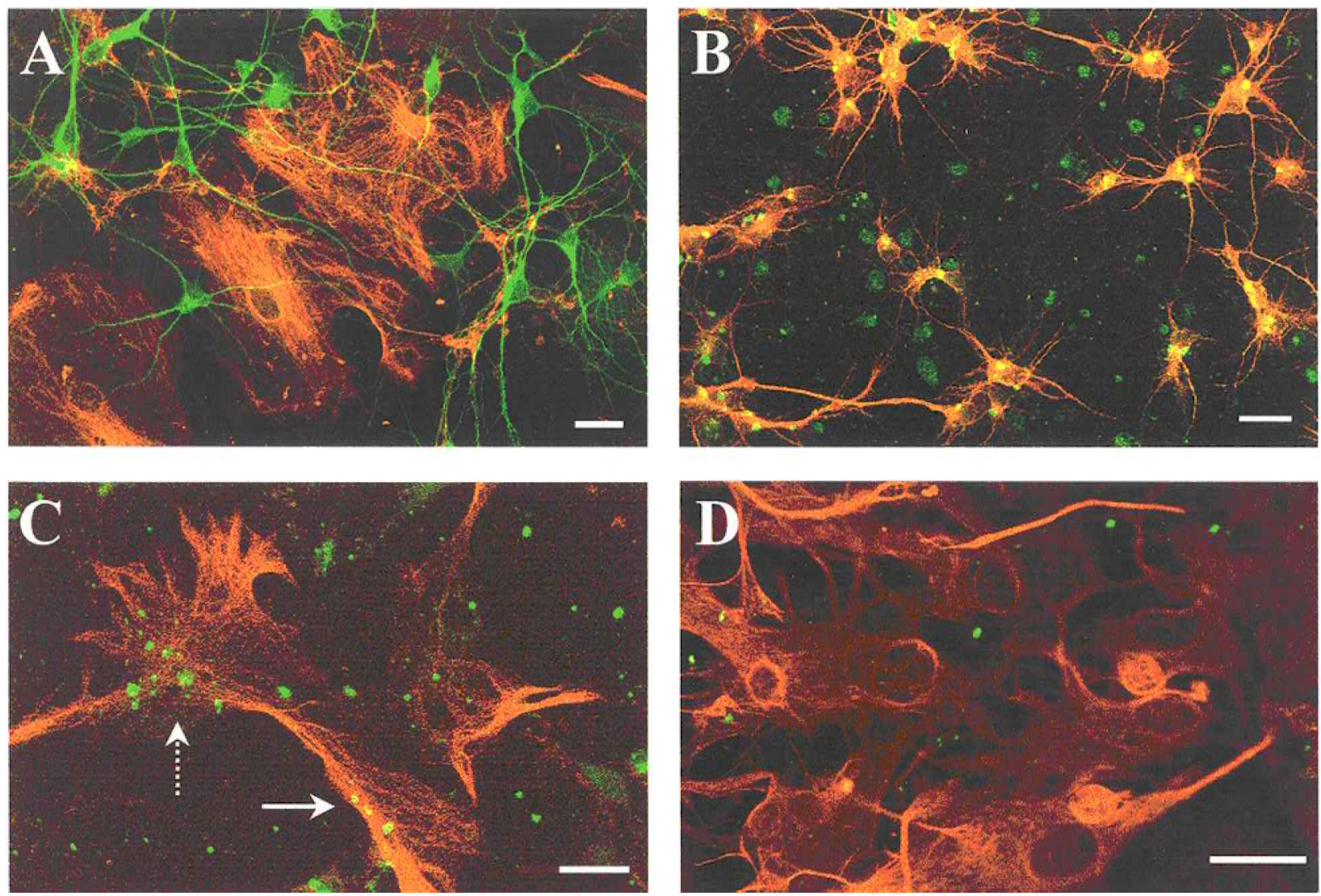

Figure 2 Astrocytes are not permissive to RV infection in vitro. (A) Neurons and astrocytes were detected in dissociated embryonic SC cultures after 14 days in vitro with anti-MAP2 (green) and anti-GFAP (red) antibodies, respectively. (B) In these cultures, 24 h after infection with CVS, infected cells were detected with an anti-nucleocapsid antibody (green). All the MAP2 immunoreactive neurons (red) contained the viral antigen, as indicated by the yellow staining present in their soma. (C) In the same culture, GFAP immunoreactive cells (red) contained rarely the viral antigen (yellow, designated by a full arrow). The superimposition of the two labelings (green, indicated by a dotted arrow) suggest the presence of infected GFAP ${ }^{-}$cells above a noninfected $\mathrm{GFAP}^{+}$cell. (D) In astrocytes cultured for 14 days and infected with CVS for 3 days, a faint viral labeling (green) is separated from GFAP immunoreactivity (red), indicative of an absence of multiplication of RV in astrocytes. All images were collected as single optical sections by CSLM after excitation at 488- and 543-nm wavelengths. Overlays were generated after superimposition of single color images. Bars $=25 \mu \mathrm{m}$.

these neurons were infected under different culture conditions: incubation with conditioned medium collected from RV-infected SC cultures (replaced every 2 days) to detect a diffusible factor responsible for the MNs' death. Also, coculture of MNs together with astrocytes or BSR cells to analyze the role of neighboring infected cells in the neuronal susceptibility to RV in SC cultures. None of these experimental conditions affected the survival of RV-infected MNs, at least during 3 days of infection (data not shown). Incidentally, these experiments showed that astrocytes were not infected, confirming that those cells were not susceptible to RV (Figure 2D).

\section{Virus-induced apoptosis in MNs}

To further investigate MNs' survival after RV infection, CVS-infected MNs were subjected to a TUNEL assay. This experiment allowed estimation of the

Table 1 Neuronal mortality in RV-infected spinal cord cultures

\begin{tabular}{|c|c|c|c|c|}
\hline Experiment number & Time after infection & $\begin{array}{c}\text { Neurons } / \mathrm{mm}^{2} \text { in } \\
\text { uninfected cultures } \\
\left(\% \pm \text { s.e.m. }{ }^{*}\right)\end{array}$ & $\begin{array}{l}\text { Neurons } / \mathrm{mm}^{2} \text { in } \\
\text { infected cultures } \\
\quad(\% \pm \text { s.e.m.) }\end{array}$ & $\begin{array}{l}\text { Virus-induced } \\
\text { mortality } \\
(\%)\end{array}$ \\
\hline 1 & 2 days & $109.0 \pm 21.5$ & $31.7 \pm 14.2$ & 70.9 \\
\hline 2 & 2 days & $34.0 \pm 2.6$ & $9 \pm 3.6$ & 73.5 \\
\hline 3 & 3 days & $110.3 \pm 4.2$ & $19.7 \pm 3.2$ & 82.1 \\
\hline
\end{tabular}

After 14 days in vitro, the cultures were infected with CVS at a m.o.i. $>1$ for 2 or 3 days or mock-infected. Neurons were detected by immunocytochemistry with an anti-MAP2 antibody and MAP2 $2^{+}$cells were counted at a $400 \times$ magnification in fields of 1 mm ${ }^{2}$ area. $n \geq 3$. *s.e.m.: standard error of the mean. 

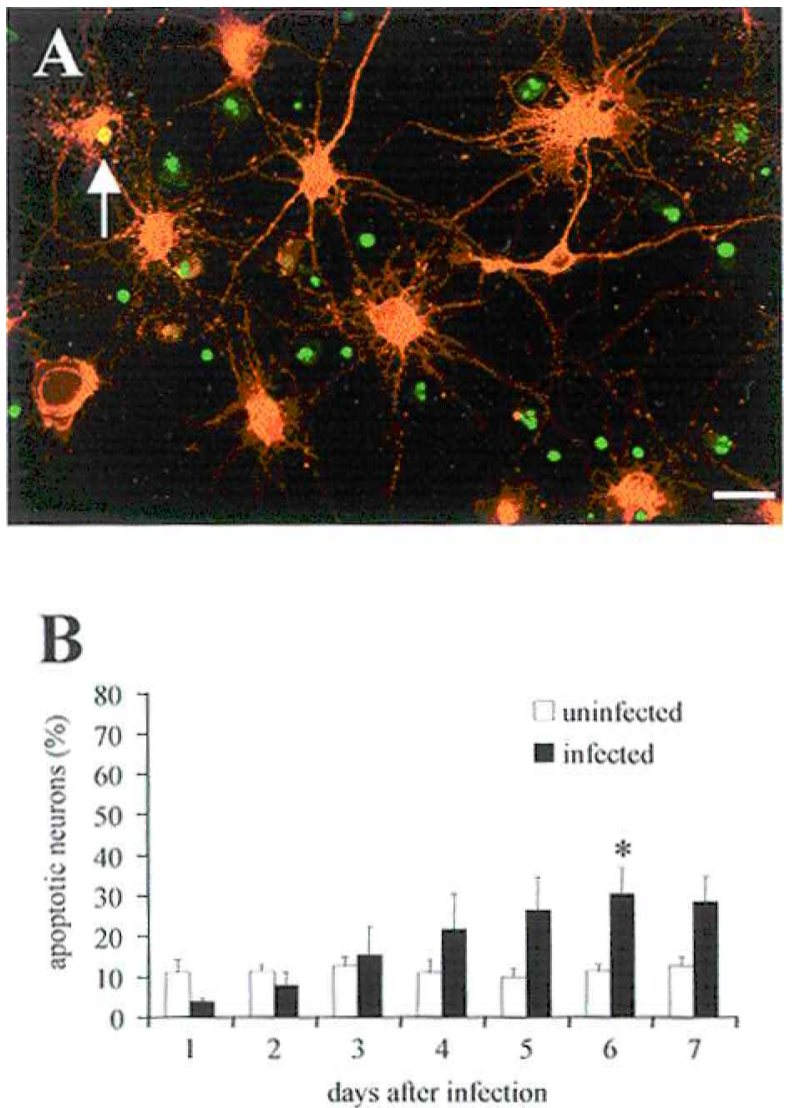

Figure 3 Low level of apoptosis in MNs after infection by RV in vitro. (A) Purified MNs were infected for 7 days with RV. After fixation and permeabilization, infected neurons were detected with an anti-rabies phosphoprotein antibody (red) and the apoptotic cells were identified by the TUNEL method as described in materials and methods (green). In this field, only one infected neuron was apoptotic (yellow nucleus indicated by the arrow). The images were collected as single optical sections by CSLM at the two different wavelengths. The overlay was generated after superimposition of single color images. Bar $=25 \mu \mathrm{m}$. (B) Infected and noninfected purified MNs cultures were processed daily between day 1 and 7 for the simultaneous detection of infected (with an anti-RV-P antibody) or noninfected (with an anti-MAP2 antibody) neurons and apoptosis (by TUNEL assay). The percentage of apoptotic neurons was determined by counting of the double-labeled cells among infected and noninfected cultures. Asterisks indicate values in infected cultures that are statistically significant at $P<$ 0.05 by Student's $t$-test compared to that obtained in uninfected cultures. Error bars represent the standard error of the mean. $n=4$.

percentage of apoptotic cells among the infected neurons. The results presented in Figure $3 \mathrm{~A}$ indicated that only a few infected MNs exhibited a TUNEL positive signal in their nucleus. This confirmed the survival capacity of these cells following infection with RV, as already observed in the morphological analysis. In every culture submitted to the TUNEL assay (infected or uninfected), a large number of nuclei presented a strong signal of apoptosis (green labeling in Figure 3A). They belonged to cells devoid of viral labeling, which were considered morphologically as dead (the trypan blue exclusion assay was incompatible with the TUNEL assay). These cells cor- responded to motoneuron corpses, probably already present as early as the beginning of the culture, because the percentage of living MNs remained stable during the course of the experiment. During the first 7 days following infection of MNs with RV, we quantified the number of infected neurons that gave a positive signal in the TUNEL assay. The results presented in Figure 3B indicated that no more than $30 \%$ of $\mathrm{MNs}$ were apoptotic at 7 days p.i., yet around $10 \%$ showed DNA fragmentation in non-infected MNs. This difference was not considered as significant using the unpaired two groups Student's $t$-test $(P<0.05)$.

In vivo experiments were conducted on RVinfected rat neonates in order to see whether MNs were apoptotic in the later stages of the infection. Four days after unilateral injection of a high dose of CVS in the hind limb, infection was massive in the lumbar spinal cord (Figure 4A). A TUNEL assay applied on an adjacent section showed the presence of apoptotic nuclei in the ipsilateral ventral horn of the spinal cord, suggesting that some of them could correspond to MNs (Figure 4B). The density of labeling in this area corresponded to $30 \%$ of the density observed in the positive control (data not shown) suggesting that only part of the cells accessible to the TUNEL reaction were apoptotic. In contrast, on the contralateral side, only rare nuclei were labeled, indicating that most of the MNs were not apoptotic (Figure 4C).

\section{Neurite growth in MNs infected with $R V$}

All these results led to the conclusion that MNs were a neuronal population surviving following RV infection in vitro. This result was unexpected, because $\mathrm{RV}$ was known to induce a light but observable CPE in nonneuronal cells (i.e., in BSR cells used to produce CVS stocks). To estimate the impact of viral infection on cellular metabolism, we compared, by quantitative morphometry, the growth of the longest neurite (that can be considered as the axon) in infected and noninfected MNs during 7 days. A previous study had shown that, among several morphometric criteria, the axon length was the parameter that increased the most dramatically during 7 days of culture (Bataillé et al, 1998). Individual MNs in low density cultures, infected or noninfected with RV, were photographed daily during 7 consecutive days and the length of their longest neurite was measured (Figure 5A). These data are presented in Figure 5B. They showed that, despite their infection with RV, MNs developed an axon as efficiently as the noninfected ones. This result strongly suggests that, under our experimental conditions, the metabolism of MNs was not perturbed by RV infection during this period of time.

\section{Hippocampus neurons are susceptible to $R V$ infection}

To check whether the survival of RV-infected neurons was dependent on the nature of the neuronal 

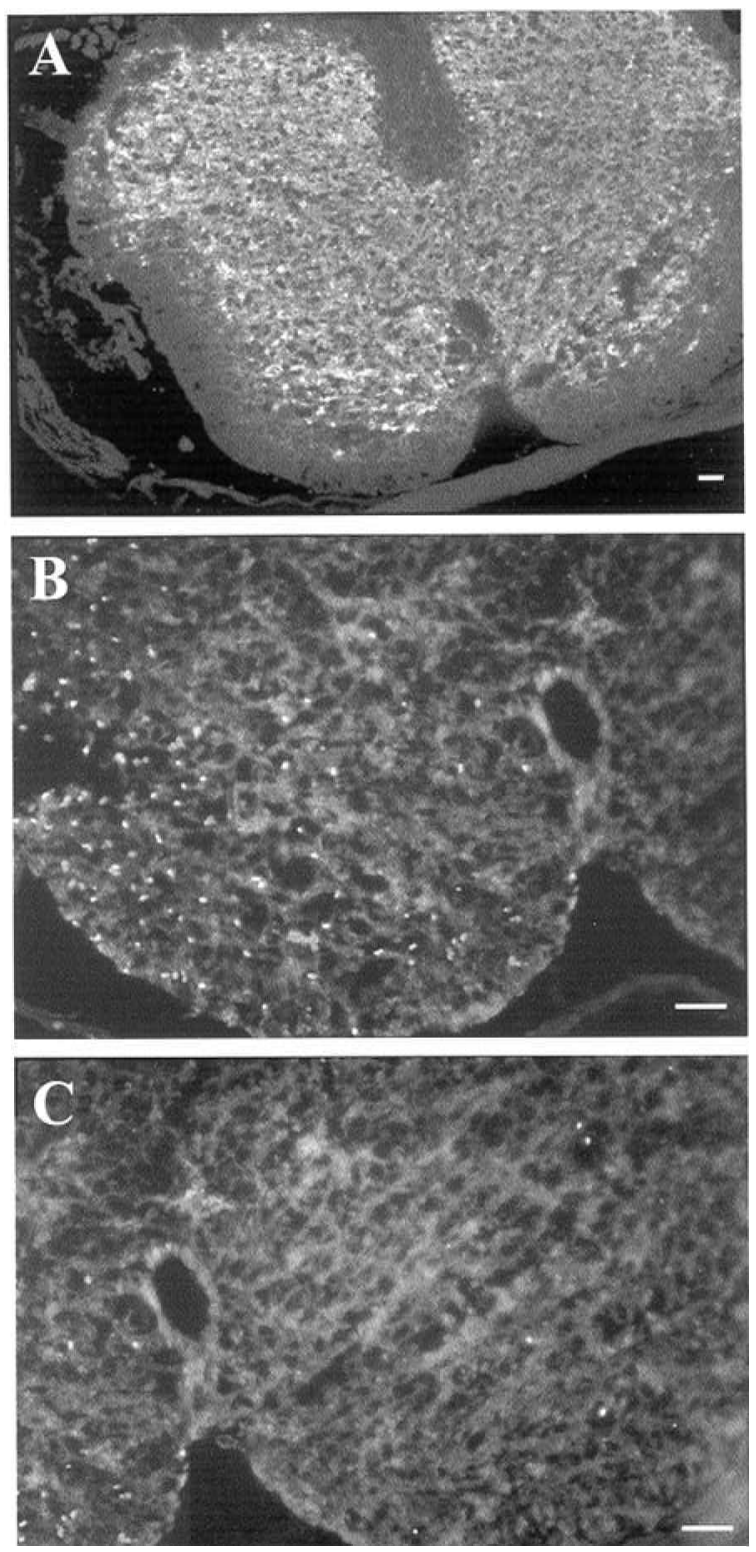

Figure 4 Apoptosis in the spinal cord of rat neonates infected with RV. (A) Immunohistochemical detection of RV-infected neurons in the lumbar spinal cord of a rat pup, 4 days after hind limb inoculation. The ipsilateral side corresponded to the left part of the cord. (B and C) On the adjacent section, apoptosis was detected by the TUNEL method and fragmented DNA containing cells were identified by white dots in the ipsilateral (B) and the contralateral (C) part of the cord. Bar $=100 \mu \mathrm{m}$.

population, infections were performed on embryonic hippocampus neurons purified using the procedure applied for the isolation of MNs (see materials and methods). Under these conditions, purified cultures were enriched in pyramidal neurons and were free of glial cells in most cases (Figure 6A). The results showed that hippocampus neurons were destroyed by RV infection, because less than $10 \%$ of these neurons were still present in the cultures after 3 days of infection (Figure 6B). Hippocampus cultures were

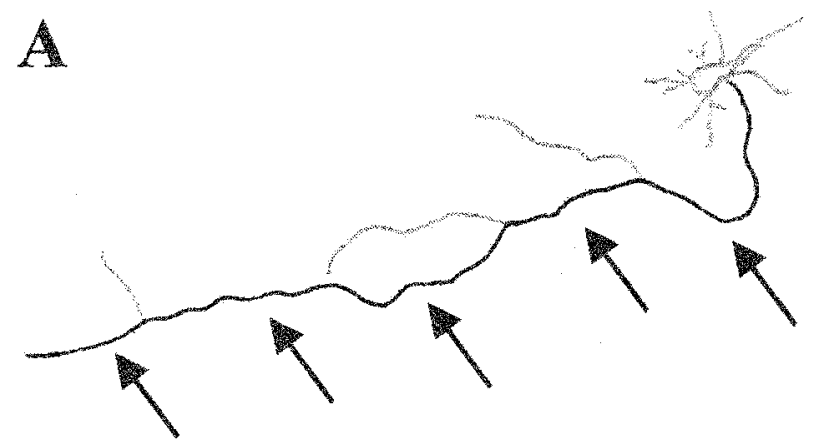

B

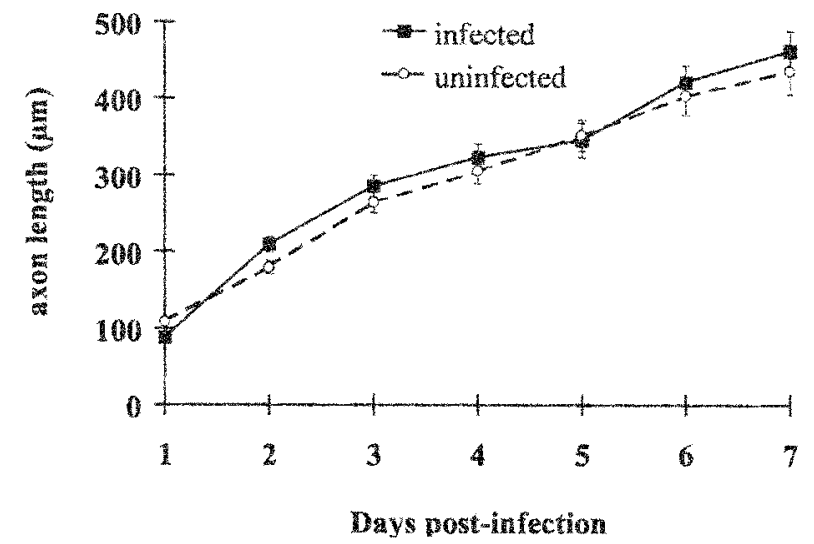

Figure 5 Unaffected growth of axons in CVS-infected MNs in vitro. Purified MNs were infected or not with RV for 1 to 7 days. Daily, the length of their axon was measured under both conditions from phase contrast pictures, as described in materials and methods. (A) Drawing of an infected MNs at 4 days p.i. where the main branch of the axon (in black) is indicated by arrows. (B) The axonal length was measured on $30 \mathrm{MNs}$ at each time. The size of these axons increased constantly during 7 days after infection of MNs. No significant changes were observed. Error bars represent the standard error of the mean. $n=3$.

submitted to a TUNEL assay, in order to estimate the percentage of apoptotic cells among infected and uninfected neurons. We observed that almost $50 \%$ of infected neurons were apoptotic at $48 \mathrm{~h}$ p.i. and $70 \%$ at $72 \mathrm{~h}$ p.i. (Figure 6C). This result is in accordance with the high mortality observed in infected hippocampus neurons as early as $48 \mathrm{~h}$ p.i. (Morimoto et al, 1999).

On the other hand, because purified MNs (and not SC and hippocampus cultures) were grown in presence of inserts of myoblasts (see materials and methods), we investigated the possible protective effect of factors secreted by muscular cells on the survival of hippocampus neurons infected with RV. The monitoring of infected neurons cultured with or without inserts of myoblasts showed no difference in their capacity to survive RV infection (data not shown), indicating that myoblasts did not secrete molecules having a protective effect on hippocampus neurons against RV infection. 

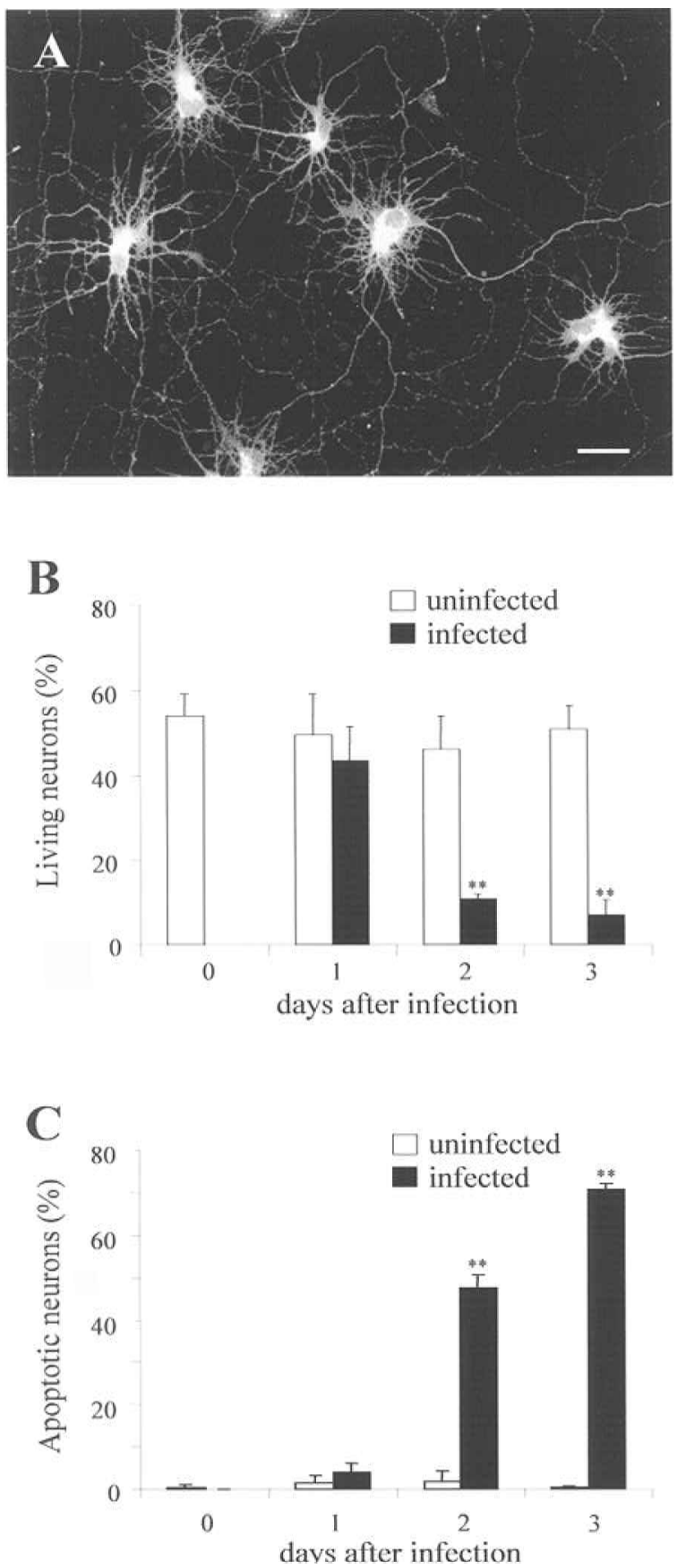

Figure 6 Survival of hippocampus neurons after rabies virus infection in vitro. (A) Purified hippocampus neurons after 8 days in vitro were infected with CVS for $24 \mathrm{~h}$. Detection with an anti-P MAb. The image was collected as a single optical section by CSLM after excitation at 543-nm wavelength. Bar $=25 \mu \mathrm{m}$. (B) Infected and noninfected purified hippocampus cultures were photographed daily and the percentage of living neurons was estimated in each preparation during 3 consecutive days using a morphological criterion. Double asterisks indicate values in infected cultures that are statistically significant at $P<0.01$ by Student's $t$-test compared to that obtained in uninfected cultures. Error bars represent the standard error of the mean. $n=4$. (C) Infected and noninfected purified hippocampus cultures were processed daily between day 1 and day 3 for the simultaneous detection of infected (with an anti-RV-P antibody) or noninfected (with an anti-MAP2 antibody) neurons and apoptosis (by TUNEL assay).

\section{Discussion}

In this study, primary cultures of purified rat MNs have been used to investigate neuronal infection with $\mathrm{RV}$ in vitro. Morphological observations of infected MNs (Figure 1A-B) indicated that cultured at low density, these neurons survived in vitro for at least 7 days (Figure 1C). At the same time, infected MNs did not show major signs of apoptosis since only $30 \%$ of them presented chromosomal DNA fragmentation, as detected by TUNEL staining compared to $10 \%$ observed in uninfected MNs (Figure 3A-B). Besides, this increase in the number of apoptotic MNs was not considered as statistically significant. In vivo experiments using rat neonates showed a moderate level of apoptosis in the spinal cord of RV-infected animals after 4 days of infection, especially in the ventral horn (Figure 4B), compared to the quantity of infected neurons (Figure 4A). Most of the apoptotic nuclei were found ipsilaterally to the injection site (compare Figures $4 \mathrm{~B}$ and $4 \mathrm{C}$ ), suggesting a relation between the level of apoptosis and the time of the infection, because contralateral neurons were infected later than ipsilateral ones.

Some of the apoptotic neurons could be MNs but this could not be definitely demonstrated in absence of both immunohistochemical identification of RVinfected MNs and TUNEL assay on the same section. In any case, the number of apoptotic MNs would probably be low. These results would appear to be in contradiction with previous published data. Thus, there are numerous examples showing that neurotropic viruses may cause neuronal cell death by apoptosis. This concerns both DNA viruses, such as herpes viruses (Ozaki et al, 1997), and RNA viruses, either enveloped such as alphaviruses (Lewis et al, 1996; Després et al, 1998), bunyaviruses (Pekosz et al, 1996) and paramyxoviruses (Evlashev et al, 2000) or unenveloped such as picornaviruses (Tsunoda et al, 1997; Girard et al, 1999) and reoviruses (Oberhaus et al, 1996). At the same time, evidence has been offered that this programmed cell death mechanism is probably also induced in neurons following RV infection, both in vivo (Jackson and Rossiter, 1997; Jackson and Park, 1998) and in vitro (Morimoto et al, 1999). This latter study described the survival of mouse embryonic hippocampal neurons after infection with two variants of the strain CVS-24: CVS-B2c (multiplied in BHK-21 cells) and CVS-N2c (multiplied in neuroblastoma cells) differing from each other by 10 amino acids in the $G$ protein (Morimoto et al, 1998). After infection with CVS-B2c, the neurons begin to degenerate at $72 \mathrm{~h}$ p.i. while they are resistant to infection

The percentage of apoptotic neurons was determined by counting of the double labeled cells among infected and noninfected hippocampus cultures. Double asterisks indicate values in infected cultures that are statistically significant at $P<0.01$ by Student's $t$-test compared to that obtained in uninfected cultures. Error bars represent the standard error of the mean. $n=3$. 
by CVS-N2c (Morimoto et al, 1999). This analysis was not taken any further.

It has been known for a long time that RV-G plays a key role in the pathogenesis of rabies (Coulon et al, 1994). It happens that amino acid $G$ sequences of CVS-B2c and the CVS strain used in this study are strictly identical (Préhaud et al, 1988; Morimoto et al, 1998), suggesting that the differences in the survival capacities of rat MNs and mouse hippocampal neurons with regard to RV infection did not originate from the virus. Incidentally, in an in vitro study focused on the entry of RV into neurons, the authors reported that rat embryonic hippocampal neurons did not survive CVS infection for more than 4 days (Lewis and Lentz, 1998). It should be noted that the viral strain used in these experiments was grown in neuroblastoma cells, making it probably similar to CVS-N2c, considered as a low cytopathic virus for neurons (Morimoto et al, 1999). Our in vitro results confirmed that hippocampus neurons were sensitive to RV infection since more than $90 \%$ of CVS-infected hippocampus neurons were destroyed within 3 days (Figure 6B). Likewise, the high percentage of apoptotic neurons in those cultures (Figure 6C) corroborate in vivo observations that indicated the pyramidal neurons of the hippocampus were the first apoptotic neurons observed in CVS-infected mouse brain after intracerebral inoculation (Jackson and Rossiter, 1997).

An experiment carried out to estimate the level of activity of the neuronal metabolism demonstrated that the longest neurite (considered as the axon) grew at the same rate in infected and uninfected MNs during 7 days (Figure 5B). At first sight, this result might be contradictory in comparison with published data. Indeed, a recent study focusing on the gene expression in RV-infected mouse brain indicated that around $90 \%$ of cellular genes were downregulated by a 4-fold lower level after 6 days of infection with CVSN2c, while only $1.6 \%$ were upregulated (Prosniak et al, 2001). This result reflected the general behavior of neuronal and nonneuronal populations, but it did not take into account minority groups of neurons that would not be affected in their gene expression after RV infection. Our morphometry analysis indicates that the cellular machinery used in axonal growth is still functional in RV-infected MNs, which supports the assumption that a strong cellular activity is maintained in infected MNs despite the synthesis of large quantities of viral proteins. At a functional level, RV infection acts differently on membrane currents in cells of the neuronal lineage. Thus, it has been shown to reduce the voltage-dependent $\mathrm{Na}^{+}$current and inward rectifier $\mathrm{K}^{+}$current in mouse neuroblastoma NA cells, while the delayed rectifier $\mathrm{K}^{+}$current was not altered (Iwata et al, 1999). In the same way, the voltage-dependen $\mathrm{Ca}^{2+}$ current was not modified in $\mathrm{RV}$-infected mouse neuroblastoma $\times$ rat glioma hybrid NG108-15 cells (Iwata et al, 2000). Such analyses have not been included in this program, but they would provide valuable information on the evolution of electric properties of MNs during RV infection.

However, this capacity to survive RV infection was not observed when MNs were cocultured with other cell types present in the spinal cord. In that case, infection with CVS resulted in the rapid death of all the neurons (Table 1). Two broad hypotheses could be offered to explain this result. In the first hypothesis, RVinfected MNs would be protected from apoptosis by factor(s) secreted by muscular inserts present at the vicinity of the MNs cultures during the course of the infection (see methods). Infection of MNs cultured in absence of myoblasts did not reduce their survival capacity compared to uninfected MNs (see results). Correlatively, the addition of inserts of myoblastic cells in the CVS-infected hippocampus cultures did not increase the survival of these neurons after RV infection. Both results indicate no protective effect of muscle cells on RV-infected neurons. In the second hypothesis, a factor would be responsible for MNs death in SC cultures. This factor could be diffusible, such as proteases released after the lysis of other infected cells, because numerous cells other than neurons were found to be infected in CVS-infected SC cultures (Figure 2B, green labeling). This hypothesis is probably not true, because CVS-infected purified MNs survived when cultured in presence of supernatant collected from infected SC cultures. This factor responsible for the neuronal death could be not diffusible and a physical contact would be necessary between an infected MNs and another infected cell to induce the programmed cell death process. This putative cell would not be an astrocyte because astrocytes are clearly nonpermissive to RV (Figure 2D), even if the idea that uninfected astrocytes might play a role in neuronal cell death could not be rejected. Moreover, not all infected cells could play this role since, in BSR-MNs cocultures infected with CVS, MNs survived at least 3 days on monolayers of infected BSR cells. Other SC neurons different from MNs could be suspected of the deleterious effect because they may form synapses with MNs, but this assumption has not been tested.

Taken together, our results argue for a strong capacity of MNs to survive RV infection. Interestingly, they can be compared to recent observations emphasizing that MNs behaved as a particular neuronal population towards a viral infection. Indeed, in mice infected with a neuroadapted strain of Sindbis virus, lumbar MNs degenerate but this loss is not apoptotic unlike the neuronal death observed in the brain of Sindbis-infected animals (Havert et al, 2000). Our in vitro study of MNs infection with RV corroborates this previous observation. MNs infected with RV induces only a light CPE and these neurons are not engaged in an apoptotic process.

At least one other neuronal population showed a similar resistance to RV infection. In vitro experiments performed using rat embryonic dorsal root ganglia (DRG) showed that these ganglionic neurons 
infected with CVS survived at least 4 weeks in culture (Lycke and Tsiang, 1987). This result indicated a total absence of CPE in these infected cultures, leading the authors to offer the hypothesis of the intrinsic resistance of ganglionic neurons to RV infection (Lycke and Tsiang, 1987). Detection of DNA fragmentation in infected neurons was not performed during this study, but it can be assumed that DRG neurons were not apoptotic during the course of the infection.

In conclusion, our in vitro results offer evidence that all neuronal cell types do not react similarly to RV infection. Their varying susceptibility can be explained by the delay in the induction of apoptosis. Because this programed neuronal cell death mechanism is delayed in MNs, these neurons are not destroyed by infection with RV. Consequently, it can be assumed that hippocampus neurons died mainly following the apoptotic process, rather than from the viral infection itself. Finally, our observation s suggest that the paralyses frequently observed in rabid animals could be a consequence of dysfunctions in the locomotor network or in MNs themselves. These functional disorders could be analyzed in vitro in MNs cultures.

\section{Materials and methods}

\section{Cells and virus}

BSR cells (a clone of BHK-21 cells) were grown in minimal essential medium (MEM; GIBCO-BRL) supplemented with $8 \%$ calf serum (Bio-Wittaker) at $37^{\circ} \mathrm{C}$ in a $5 \% \mathrm{CO}_{2}$ incubator.

The CVS strain of RV used in this study originated from the Laboratoire de Génétique des Virus, CNRS, Gif sur Yvette, France. It is genetically related to the CVS-11 strain (Smith et al, 1992). It was multiplied by infecting BSR cells at a multiplicity of infection (MOI) of 0.1 plaque forming units (PFU) per cell. After $72 \mathrm{~h}$ of infection at $37^{\circ} \mathrm{C}$ in MEM supplemented with $2 \%$ calf serum, supernatants were collected, centrifuged at a low speed, and frozen in aliquots at $-80^{\circ} \mathrm{C}$. Virus particles were concentrated according to Gaudin et al (1992). The titer of viral suspension was determined as already described (Raux et al, 1995).

\section{In vivo infection of rat neonates}

One-day-old Wistar rat pups (Centre d'élevage et de Recherche Janvier, France) were injected intramuscularly into the hind limb with $8 \times 10^{6}$ plaque-forming units (PFU) of CVS under $2 \mu \mathrm{l}$. Four days later, when they presented typical signs of rabies, the animals were perfused intracardially with $4 \%$ paraformaldehyde in phosphate-buffered saline (PBS). The vertebral column was decalcified in PBS $+10 \%$ EDTA during 7 days, then cryo-preserved in $20 \%$ sucrose for $24 \mathrm{~h}$. The tissue was frozen at $-80^{\circ} \mathrm{C}$ until use.

\section{Preparation of cultures of crude spinal cord} or of purified MNs from rat embryos

The cultures were prepared from pregnant Wistar rats at 15 days of gestation (Centre d'Élevage et de
Recherche Janvier, France) according to the technique described by Bataillé et al (1998). The first steps were common for both preparations. The pregnant rat was euthanised and embryos were removed under sterile conditions. The spinal cords were dissected and kept in calcium- and magnesium-free PBS (PBS ${ }^{-}$; GIBCO-BRL). Following removal of the meninges, spinal cords were placed in fresh $\mathrm{PBS}^{-}$and cut in small pieces. Spinal cord fragments were incubated in PBS $^{-}$containing trypsin $(0.025 \%$; Sigma $)$ and DNase I (0.005\%; Roche Molecular Biochemicals) for $30 \mathrm{~min}$ at $37^{\circ} \mathrm{C}$. After centrifugation at $700 \times$ $\mathrm{g}$ for $10 \mathrm{~min}$, the cells were dissociated through a siliconized Pasteur pipette and incubated in $\mathrm{PBS}^{-}$containing DNase I $(0.001 \%)$ for $10 \mathrm{~min}$ at $37^{\circ} \mathrm{C}$. After centrifugation, the pellet was resuspended in $3 \mathrm{ml}$ of Hank's balanced salt solution (HBSS; GIBCO-BRL) and the cells were mechanically dissociated with firepolished siliconized Pasteur pipettes of successively decreasing internal diameters. Then, the suspension was laid on the top of a $3.5 \%$ bovine serum albumin (BSA; Roche Molecular Biochemicals) cushion in $5 \mathrm{ml}$ HBSS.

Cells were centrifuged for $10 \mathrm{~min}$ at $200 \times \mathrm{g}$ to remove debris and the pellet was resuspended in $2 \mathrm{ml}$ of Neurobasal medium (GIBCO-BRL) supplemented with B27 (2\% V/V; GIBCO-BRL), horse serum ( $2 \% \mathrm{~V} / \mathrm{V}$; GIBCO-BRL), 2-mercaptoethanol (25 $\mu \mathrm{M}$; Fluka), and gentamycine (100 $\mu \mathrm{g} / \mathrm{ml}$; Sigma) in the case of crude primary spinal cord culture preparations. The cell suspension $(100 \mu \mathrm{l})$ was seeded onto $12 \times 24 \mathrm{~mm}$ glass coverslips treated with poly-Llysine (5 $\mu \mathrm{g} / \mathrm{ml}$; Sigma). Otherwise, after the centrifugation through the BSA cushion, the pellet was resuspended in $2 \mathrm{ml}$ of HBSS to proceed with the purification of MNs. This cell suspension was placed at the top of 2 cushions of Nycoprep 1.15 (Nycomed, Oslo, Norway) in HBSS $(20 \%$ above $60 \%)$ and then centrifuged at $700 \times \mathrm{g}$ for $26 \mathrm{~min}$ at $4^{\circ} \mathrm{C}$ in a refrigerated Sigma K3 centrifuge. Cells concentrated at the HBSS-Nycoprep $20 \%$ interface were collected.

The cell suspension was diluted in HBSS and centrifuged. Purified MNs were resuspended in supplemented Neurobasal medium. Cell density was adjusted from $1.5 \times 10^{5}$ to $2 \times 10^{5}$ cells $/ \mathrm{ml}$ or $2 \times$ $10^{6}$ cells $/ \mathrm{ml}$ according to the experiment. Then, $100 \mu \mathrm{l}$ of the suspension was plated onto glass coverslips previously coated with poly-L-lysine $(5 \mu \mathrm{g} / \mathrm{ml})$ and laminin ( $2 \mu \mathrm{g} / \mathrm{ml}$; Sigma), either directly or onto BSR-coated coverslips prepared $24 \mathrm{~h}$ before. Whatever the type of cultures, coverslips were placed individually into Petri dishes $(\varnothing: 3.5 \mathrm{~mm})$ and incubated at $37^{\circ} \mathrm{C}$ in a $5 \% \mathrm{CO}_{2}$ incubator. Following a 1-h adhesion period, $2 \mathrm{ml}$ of supplemented Neurobasal medium without horse serum was added. During short duration cultures, the medium was replaced daily. When MNs were cultured over long periods of time ( $>5$ days), explants of myoblasts and ciliary neurotrophic factor (CNTF; R \& D Systems, $1 \mathrm{ng} / \mathrm{ml}$ ) were added to the culture medium. Fifty 
percent of the medium was changed every 2 or 3 days. Morphometric and immuno-cytochemical analyses performed on purified MNs preparations indicated that 90 to $95 \%$ of the cells had properties of MNs (Bataillé et al, 1998).

\section{Preparation of muscle cells}

To increase the survival capacity of purified MNs in vitro, the cells were cultured in presence of embryonic skeletal muscle cells, the natural targets of these neurons, which have been demonstrated to secrete neurotrophic factors (Henderson et al, 1993). Myoblasts were obtained from limbs of E14-E15 rat embryos. Briefly, limbs were cut in small pieces and the tissue was incubated for $30 \mathrm{~min}$ at $37^{\circ} \mathrm{C}$, in HBSS containing $0.1 \%$ of type A collagenase (Roche Molecular Biochemicals). The tissue was triturated with a siliconized Pasteur pipette. The suspension was filtered through a $10-\mu \mathrm{m}$ nylon mesh. Cells were centrifuged at $700 \times \mathrm{g}$ for $10 \mathrm{~min}$ and the pellet was resuspended in Neurobasal medium with additives. Then, $200 \mu \mathrm{l}$ of the muscle cells suspension were seeded in tissue culture inserts (porosity: $0.2 \mu \mathrm{m}$; Nunc) and the inserts were placed in the Petri dishes.

\section{Cultures of glial cells}

These were prepared from cortex of 1- to 2-day postnatal rat pups. After dissection and cutting into small pieces, the tissue was enzymatically digested with trypsin $\left(0.025 \%\right.$; Sigma) in $\mathrm{PBS}^{-}$for $30 \mathrm{~min}$ at $37^{\circ} \mathrm{C}$. Then the cells were triturated with a siliconized Pasteur pipette and they were passed through a $130-\mu \mathrm{m}$ nylon mesh. After centrifugation of $10 \mathrm{~min}$ at $200 \times$ $\mathrm{g}$, the pellet was resuspended in Dulbecco's modified essential medium (DMEM, GIBCO-BRL) supplemented with $10 \%$ fetal calf serum (GIBCO-BRL) at a concentration of $1.5 \times 10^{6}$ cells $/ \mathrm{ml}$. The cells were distributed in $75-\mathrm{cm}^{2}$ flasks $(10 \mathrm{ml} /$ flask $)$ and the medium was changed every 2 or 3 days. When confluent, the cells consisting only in glial cells were trypsinized, then seeded onto poly-L-lysine + laminin-treated coverslips placed in Petri dishes. One week later, purified MNs (see previously) were plated on this monolayer and the coculture was maintained for 2 weeks in supplemented Neurobasal medium.

\section{Primary cultures of hippocampus neurons}

The hippocampus was dissected from E20-E21 rat embryos. After enzymatic and mechanical dissociations of the tissue, hippocampal neurons were prepared strictly according to the same protocol used for MNs purification. The centrifugation through the Nycoprep cushions separated large neurons from small interneurons and nonneuronal cells. Thus, the population recovered at the HBSS-Nycoprep 20\% interface was enriched in pyramidal neurons and was free of glial cells. The neurons were seeded onto poly-Llysine + laminin-treated coverslips at a density of 1.5 to $2 \times 10^{4}$ cells/coverslip.

\section{Infection of neurons}

After various times of culture, the neurons were infected with CVS as follows. The volume of medium was reduced to $1 \mathrm{ml}$ in the Petri dish and $0.1 \mathrm{ml}$ of cell culture supernatant diluted in Neurobasal medium was added in order to adjust the viral inoculum to $10^{6} \mathrm{UFP} /$ dish. At that concentration, close to $100 \%$ of MNs were infected. After a 30-min adsorption period at $37^{\circ} \mathrm{C}$, the inoculum was removed and the MNs were covered with supplemented Neurobasal medium and incubated for different times of infection at $37^{\circ} \mathrm{C}$ in a $5 \% \mathrm{CO}_{2}$ incubator.

\section{Antibodies}

All the antibodies used in this study were diluted in PBS. The anti-rabies nucleocapsid antibody conjugated to fluorescein isothiocyanate (FITC, Sanofi) was used at a 1/40 dilution. The anti-rabies phosphoprotein mouse monoclonal antibody $31 \mathrm{G} 10$ was isolated and characterize d at the Laboratoire de Génétique des Virus (CNRS, Gif/Yvette, France) (Raux et al, 1997). It was used as a hybridoma supernatant diluted at 1/10 or an ascitic fluid diluted at $1 / 3000$. The mouse antimicrotubule associated protein monoclonal antibody (MAP2, Sigma) was diluted at 1/300. The goat antiglial fibrillary protein antibody (GFAP, Santa Cruz Biotechnology) was used at a 1/300 dilution. All the secondary antibodies coupled either to FITC, tetramethyl rhodamine isothiocyanate (TRITC) or Texas red (TR) were purchased from Jackson ImmunoResearch Laboratories. They were used at a 1/200 dilution. In the case of double immunolabeling experiments, they were depleted of antibodies likely to cross-react with immunoglobulins of the animal species used for the preparation of the other primary antibody.

\section{Immunocytochemistry}

The cultures were fixed in paraformaldehyde $4 \%$ in PBS during $10 \mathrm{~min}$, then in paraformaldehyde $4 \%+$ Triton X-100 $0.1 \%$ in PBS for $5 \mathrm{~min}$. After three washes in PBS, primary antibodies were applied on the cells overnight at $4^{\circ} \mathrm{C}$. The cultures were washed three times in PBS and they were incubated with secondary antibodies for $4 \mathrm{~h}$ at $37^{\circ} \mathrm{C}$. After three subsequent washes in PBS, coverslips were mounted with Immu-mount (Shandon) and observed under a BX50 Olympus UV microscope equipped with UV filters.

\section{Image analysis}

Confocal scanning light microscopy (CSLM) analyses of double-stained cultures were performed with a Zeiss LSM 410 apparatus. From a given field, two separate images were obtained as optical sections after excitation at $488 \mathrm{~nm}$ (green labeling) and $543 \mathrm{~nm}$ (red labeling). These two images were merged to generate a composite picture in which a yellow staining was indicative of the vicinity of FITC and TRITC fluorochromes. 


\section{Estimation of the neuronal viability}

The cultures were observed with an inverted microscope equipped with phase contrast (IMT2, Olympus). In each culture condition, daily, 10 fields were randomly selected and they were photographed with a video CCD camera (Sony) coupled to a videographic printer (Sony). Analyses were performed on video images. A neuron was considered as alive when its soma was uniformly dark. When the cell body contained one bright inclusion, a neuron was considered as dead. In selected experiments, parallel counts were performed directly under the microscope on cultures stained with trypan blue. The experiments were repeated four times and the results were expressed as the mean \pm SEM.

\section{Detection of apoptosis}

At variou s times after infection, CVS-infected and uninfected control cultures were fixed, permeabilized, and treated for the detection of neurons using the anti-rabies phosphoprotein or the anti-MAP2 antibody as primary antibody, respectively, and an antimouse IgG coupled to TRITC as secondary antibody (see previously). Then, apoptotic cells were identified by the terminal deoxynucleotidyl transferasemediated dUTP nick end labeling (TUNEL) technique (Roche Molecular Biochemicals), according to the manufacturer's prescriptions.

For in vivo experiments, CVS-infected vertebral columns were sectioned in a cryostat and $10-\mu \mathrm{m}$ consecutive sections were collected on alternate gelatinized slides. Pair sections were permeabilized and

\section{References}

Astic L, Saucier D, Coulon P, Lafay F, Flamand A (1993). The CVS strain of rabies virus as transneuronal tracer in the olfactory system of mice. Brain Res 619: 146-156.

Bataillé S, Portalier P, Coulon P, Ternaux J-P (1998). Influence of acetylcholinesterase on embryonic spinal rat motoneurones growth in culture: A quantitative morphometric study. Eur J Neurosci 10: 560-572.

Castellanos JE, Castaneda DR, Velandia AE, Hurtado H (1997). Partial inhibition of the in vitro infection of adult mouse dorsal root ganglion neurons by rabies virus using nicotinic antagonists. Neurosci Lett 229: 198-200.

Castellanos JE, Martinez M, Acosta O, Hurtado H (2000). Nerve growth factor and neurotrophin-3 modulate the rabies infection of adult sensory neurons in primary cultures. Brain Res 871: 120-126.

Coulon P, Lafay F, Tuffereau C, Flamand A (1994). The molecular basis for altered pathogenicity of lyssavirus variants. In Current Topics in Microbiology and Immunology, vol 187. Rupprecht CE, Dietzschold B, Koprowski H (eds). Springer Verlag: Berlin, pp 69-84.

Coulon P, Ternaux JP, Flamand A, Tuffereau C (1998). An avirulent mutant of rabies virus is unable to infect motoneurons in vivo and in vitro. J Virol 72: 273-278. treated for the detection of infected neurons using the anti-rabies phosphoprotein antibody and an anti-mouse IgG coupled to TR. Unpaired sections were treated with $10 \mu \mathrm{g}$ of proteinase K (Sigma) in $10 \mathrm{mM}$ Tris-HCl (pH 7.4), $2 \mathrm{mM} \mathrm{CaCl}_{2}$ for $30 \mathrm{~min}$ at $37^{\circ} \mathrm{C}$. After two washes in PBS, sections underwent the TUNEL reaction during $1 \mathrm{~h}$ at $37^{\circ} \mathrm{C}$ to detect apoptotic nuclei. Positive and negative controls were performed on sections from uninfected animals, previously treated or not with DNAse I (Roche Molecular Biochemicals) at $1 \mathrm{mg} / \mathrm{ml}$ in $50 \mathrm{mM}$ Tris $\mathrm{HCl}$ (pH 7.4), $1 \mathrm{mM} \mathrm{MgCl}_{2}, 10 \% \mathrm{BSA}$, during $30 \mathrm{~min}$ at $37^{\circ} \mathrm{C}$.

\section{Morphometric analysis}

The axonal length was measured on infected and noninfected MNs cultures as described in Bataillé et al (1998). Briefly, these measurements were taken daily during 7 days from video images, using a graphic tablet (Summasketch III, Summagraphics) coupled to a computer. Direct measurements of length were performed using morphometric software designed at University College, London. Under each condition, at least 30 cells were randomly selected and the experiment was repeated three times. Results were expressed as the mean \pm SEM.

\section{Statistical analysis}

Statistical analysis was done with the unpaired two group Student's $t$-test. Values for RV-infected and uninfected conditions were compared. $P$ values $<0.05$ were considered significant.
Després P, Frenkiel MP, Ceccaldi PE, DosSantos CD, Deubel $\mathrm{V}$ (1998). Apoptosis in the mouse central nervous system in response to infection with mouse-neurovirulent dengue viruses. J Virol 72: 823-829.

Evlashev A, Moyse E, Valentin H, Azocar O, TrescolBiemont MC, Marie JC, Rabourdin-Combe C, Horvat B (2000). Productive measles virus brain infection and apoptosis in CD46 transgenic mice. J Virol 74: 13731382.

Gaudin Y, Ruigrok RWH, Tuffereau C, Knossow M, Flamand A (1992). Rabies virus glycoprotein is a trimer. Virology 187: 627-632.

Gillet JP, Derer P, Tsiang H (1986). Axonal transport of rabies virus in the central nervous system of the rat. $J \mathrm{Neu}$ ropathol Exp Neurol 45: 619-634.

Girard S, Couderc T, Destombes J, Thiesson D, Delpeyroux F, Blondel B (1999). Poliovirus induces apoptosis in the mouse central nervous system. J Virol 73: $6066-6072$.

Havert MB, Schofield B, Griffin DE, Irani DN (2000). Activation of divergent neuronal cell death pathways in different target cell populations during neuroadapted Sindbis virus infection of mice. J Virol 74: 5352-5356.

Henderson CE, Camu W, Metling C, Gouin A, Poulsen K, Karihaloo M, Rullamas J, Evans T, McMahon SB, 
Armanini M, Berkemeier L, Phillips H, Rosenthal A (1993). Neurotrophins promote motor neuron survival and are present in embryonic limb bud. Nature 363: 266-270.

Iwata M, Komori S, Unno T, Minamoto N, Ohashi H (1999). Modification of membrane currents in mouse neuroblastoma cells following infection with rabies virus. $\mathrm{Br}$ J Pharmacol 126: 1691-1698.

Iwata M, Unno T, Minamoto $\mathrm{N}$, Ohashi $\mathrm{H}$, Komori S (2000). Rabies virus infection prevents the modulation by alpha(2)-adrenoceptors, but not muscarinic receptors, of $\mathrm{Ca}^{2+}$ channels in NG108-15 cells. Eur J Pharmacol 404: 79-88.

Jackson AC, Park H (1998). Apoptotic cell death in experimental rabies in suckling mice. Acta Neuropathol 95: 159-164.

Jackson AC, Rossiter JP (1997). Apoptosis plays an important role in experimental rabies virus infection. $J$ Virol 71: 5603-5607.

Kuc̀era P, Dolivo M, Coulon P, Flamand A (1985). Pathways of the early propagation of virulent and avirulent rabies virus strains from the eye to the brain. J Virol 55: 158162.

Lafay F, Coulon P, Astic L, Saucier D, Riche D, Holley A, Flamand A (1991). Spread of the CVS strain of rabies virus and of the avirulent mutant AvO1 along the olfactory pathways of the mouse after intranasal inoculation. Virology 183: 320-330.

Lewis J, Wesselingh SL, Griffin DE, Hardwick JM (1996). Alphavirus-induced apoptosis in mouse brains correlates with neurovirulence. J Virol 70: 1828-1835.

Lewis P, Fu Y, Lentz TL (2000). Rabies virus entry at the neuromuscular junction in nerve-muscle cocultures. Muscle Nerve 23: 720-730.

Lewis P, Lentz TL (1998). Rabies virus entry into cultured rat hippocampal neurons. J Neurocytol 27: $559-573$

Lycke E, Tsiang H (1987). Rabies virus infection of cultured rat sensory neurons. J Virol 61: 2733-2741.

Morimoto K, Hooper DC, Carbaugh H, Fu ZF, Koprowski H, Dietzschold B (1998). Rabies virus quasispecies: Implications for pathogenesis. Proc Natl Acad Sci USA 95: 3152-3156.

Morimoto K, Hooper DC, Spitsin S, Koprowski H, Dietzschold B (1999). Pathogenicity of different rabies virus variants inversely correlates with apoptosis and rabies virus glycoprotein expression in infected primary neuron cultures. I Virol 73: 510-518.

Murphy FA (1977). Rabies pathogenesis. Arch Virol 54: 279-297.

Oberhaus SM, Smith RL, Clayton GH, Dermody TS, Tyler KL (1997). Reovirus infection and tissue injury in the mouse central nervous system are associated with apoptosis. J Virol 71: 2100-2106.

Ozaki N, Sugiura Y, Yamamoto M, Yokoya S, Wanaka A, Nishiyama Y (1997). Apoptosis induced in the spinal cord and dorsal root ganglion by infection of herpes simplex virus type 2 in the mouse. Neurosci Lett 228: 99-102.
Pekosz A, Phillips J, Pleasure D, Merry D, Gonzalez-Scaran o $\mathrm{F}$ (1996). Induction of apoptosis by La Crosse virus infection and role of neuronal differentiation and human bcl-2 expression in its prevention. J Virol 70: 5329-5335.

Préhaud C, Coulon P, Lafay F, Thiers C, Flamand A (1988). Antigenic site II of the rabies virus glycoprotein: Structure and role in viral virulence. J Virol 62: 1-7.

Prosniak M, Hooper DC, Dietzschold B, Koprowski H (2001). Effect of rabies virus infection on gene expression in mouse brain. Proc Natl Acad Sci USA 98: 27582763.

Raux H, Coulon P, Lafay F, Flamand A (1995). Monoclonal antibodies which recognize the acidic configuration of the rabies glycoprotein at the surface of the virion can be neutralizing. Virology 210: 400-408.

Raux H, Iseni F, Lafay F, Blondel D (1997). Mapping of monoclonal antibody epitopes of the rabies virus $\mathrm{P}$ protein. J Gen Virol 78: 119-124.

Sacramento D, Badrane H, Bourhy H, Tordo N (1992). Molecular epidemiology of rabies virus in FranceComparison with vaccine strains. J Gen Virol 73: 11491158.

Smith JS, Orciari LA, Yager PA, Seidel HD, Warner CK (1992). Epidemiologic and historical relationships among 87 rabies virus isolates as determined by limited sequence analysis. J Infect Dis 166: 296-307.

Tang Y, Rampin O, Giuliano F, Ugolini G (1999). Spinal and brain circuits to motoneurons of the bulbospongiosus muscle: Retrograde transneuronal tracing with rabies virus. J Comp Neurol 414: 167-192.

Tsiang H, Ceccaldi PE, Ermine A, Lockhart B, Guillemer $S$ (1991). Inhibition of rabies virus infection in cultured rat cortical neurons by an $N$-methyl-D-aspartate noncompetitive antagonist, MK-801. Antimicrob Agents Chemother 35: 572-574.

Tsiang H, de la Porte S, Ambroise DJ, Derer M, Koenig J (1986). Infection of cultured rat myotubes and neurons from the spinal cord by rabies virus. J Neuropathol Exp Neurol 45: 28-42.

Tsiang H, Derer M, Taxi J (1983). An in vivo and in vitro study of rabies virus infection of the rat superior cervical ganglia. Arch Virol 76: 231-243.

Tsiang H, Lycke E, Ceccaldi P-E, Ermine A, Hirardot X (1989). The anterograde transport of rabies virus in rat sensory dorsal root ganglia neurons. J Gen Virol 70: 2075-2085.

Tsunoda I, Kurtz CI, Fujinami RS (1997). Apoptosis in acute and chronic central nervous system disease induced by Theiler's murine encephalomyelitis virus. Virology 228: 388-393.

Ugolini G (1995). Specificity of rabies virus as a transneuronal tracer of motor networks: Transfer from hypoglossal motoneurons to connected second-order and higher order central nervous system cell groups. J Comp Neurol 356: $457-480$.

Wagner RR, Rose JK (1996). Rhabdoviridae: The viruses and their replication. In Fields' Virology. Fields BN, Knipe DM, Howley PM (eds). Lippincott-Raven: Philapdelphia, pp 1121-1135. 